\title{
Daniela Brogi, Un romanzo per gli occhi: Manzoni, Caravaggio e la fabbrica del realismo
}

Roma: Carocci 2018, 248 pp.

Sembra un ossimoro il titolo del saggio di Daniela Brogi, Un romanzo per gli occhi. Manzoni, Caravaggio e la fabbrica del realismo, che sottolinea come i personaggi vengono 'visti' dal narratore e come li 'vedono' i lettori. Ė subito chiaro che la letteratura romanzesca e la pittura di Caravaggio vanno di pari passo nell'indagare il mondo 'basso' degli uomini "di piccol affare", tanto rivoluzionari sia per il romanziere sia per il pittore. È tra costoro che entrambi gli artisti trovano materiale per le loro opere tanto che, rappresentandoli, elaborano narrazioni che colpiscono 'i sensi e la fantasia'.

A corredo della trattazione Brogi inserisce le immagini create dal Gonin per l'edizione della Quarantana e sottolinea il "rapporto tra parola e immagine". L'appendice riunisce tali illustrazioni e alcune riproduzioni di Caravaggio, confermando l'importanza del dialogo fra scrittura e pittura a testimonianza di quanto il legame fra parola e immagine sia fondamentale per una narrazione che raggiunga anche persone non colte, se non analfabete, come quelle a cui questi artisti si rivolgevano.

Scrive Brogi: "Lavoreremo sulla consapevolezza della spettatorialità di cui parla la cultura visuale, occupandoci però non di un testo visivo, in senso stretto, ma di un romanzo che fu così rivoluzionario e capace di funzionare come 'libro per tutti' anche grazie alla sua capacità di 'parlare agli occhi'”. Ricorda che Manzoni, scrivendo a Fauriel, spiega che nel romanzo ha voluto "rappresentare un mondo dove il consumo di narrazioni passava attraverso la cultura orale e la grammatica delle immagini, sia nelle alte sfere, che nelle basse sfere". L'ambientazione seicentesca funziona da specchio e permette di rappresentare nell'Ottocento "un immaginario radicalmente nuovo, vale a dire capace di abbassare il sacro e innalzare il basso alla dignità di racconto" e il cristianesimo costituisce il "codice culturale capace di restituire visibilità e serietà prospettica a ciò che la storia" ha fatto dimenticare. Questa 'rivoluzione' permette a tutti di capire il messaggio attraverso le illustrazioni della Quarantana come attraverso i quadri di Caravaggio.

Il cap. 3, Vivere per uno guardo: storia di Gertrude, indica l'ottica da cui è scrutato il personaggio dalla vita drammatica. Chi leggerà il capitolo dopo quelli dedicati alla monaca di Monza nel romanzo, tornerà a rileggerli e a 
riflettere su di essi con una consapevolezza ben diversa da quella del primo approccio al testo manzoniano. Il cap. 3 suggerisce quali strumenti usare per capire questo personaggio, così forte e così debole a un tempo, e il lettore si renderà conto di come proprio gli occhi (con gli sguardi che lei riceve sempre dall'alto in basso) ne condizionano le azioni per la vita intera. Imparerà il rapporto stretto tra la dimensione storica e sociale da una parte e la narrazione di una vita personale e privata dall'altra e potrà estendere questo strumento di comprensione agli altri personaggi e a tutto il romanzo.

Il sottotitolo presenta un termine imprescindibile sul quale si fonda il saggio, cioè 'realismo', che in genere applichiamo al Realismo ottocentesco in tutte le arti. Nel cap. 4, Manzoni e Caravaggio, Brogi sottolinea che nell'"universo popolare e premoderno, dove la cultura visuale era, nella maggior parte dei casi, il primo requisito di raffigurabilità e leggibilità del mondo" tale realismo è stato rivoluzionario perché "fissa e rappresenta la condizione della vita e delle cose in quanto situazioni visibili". Per le persone semplici protagoniste del romanzo hanno una grande importanza la religione e le immagini sacre che la incarnano, perché "appartengono al progetto pedagogico di una cultura popolare fatta di scambi tra racconto, immagine e parola, sotto il segno della fede e della pietà popolare". Ecco che l'opera manzoniana si fonde con quella di Caravaggio, perché "come l'arrivo improvviso della luce in molte tele di Caravaggio, il realismo manzoniano rende visibili le esistenze rimaste al buio". Infatti "il realismo è [...] stampare nelle menti, ora per via della pittura, ora per via della scrittura, gli aspetti visibili del mondo che possono risaltare dai riflessi della realtà nelle vite accidentali, immemorabili, deperibili, eppure raggiunte da un fascio di luce".

Se lo statuto epistemologico dell'arte è fissare in un' immagine o in una narrazione un momento o uno spaccato della realtà, Manzoni si serve di tutti gli oggetti che pone in scena, i quattro capponi di Renzo, "un paniere calato con una fune, un piatto di peltro", per dare un senso a quanto narra e per portare "la realtà più vicina che si può a noi, suoi lettori/spettatori" e per "far parlare il quotidiano". Ugualmente rappresenta le moltitudini, un'umanità invisibile e dimenticata, aumentando la distanza dalla storia ma anche avvicinando la vera realtà agli occhi dei lettori, come Caravaggio veste apostoli e santi con gli abiti quotidiani del suo tempo. Brogi confronta varie pagine del romanzo con quadri caravaggeschi, nei quali tutto è giocato tra luce e ombra, come nel paragone tra la Vocazione di san Matteo e il suo contraltare della 'conversione' dell'Innominato, dove la grazia e la misericordia divina 'salvano' dal peccato e dalla disperazione. Ciò che si ricava è che si deve avere fede nella redenzione, qualunque siano le prove a cui si è sottoposti.

Il cap. 2, Attraverso una natura morta, ricorda che per Manzoni il romanzo deve essere "un misto di storia e di invenzione", ottica presente sia quando i personaggi parlano dei fatti storici della guerra di successione, sia quando la guerra è rappresentata attraverso la devastazione dovuta alla carestia e alla peste, sia quando il narratore ne parla in modo critico e polemico. Il metodo manzoniano permette così di affrontare "in una prospettiva orizzontale", 
nonché di narrare e rendere comprensibili "i destini di questa umanità" altrimenti anonima.

L'ultimo capitolo, Trasparenza e opacità, giocato sull'alternanza caravaggesca luce/ombra, affronta tra l'altro il cap. XXXVIII del romanzo. Brogi porta per mano il lettore, accendendo la luce ora sui comportamenti che sembrano ripetersi, come quelli di don Abbondio, ora sul luogo di sepoltura di Azzeccagarbugli, che disorienta il lettore che si aspetta la descrizione del banchetto di nozze, ora sul non apprezzamento della bellezza di Lucia da parte dei nuovi compaesani, fino al decalogo negativo esposto da Renzo e al "sugo della storia" pronunciato dal narratore; e fa dialogare il finale con l'Introduzione, realizzando "la cornice narrativa del testo". Nella conclusione Brogi riannoda i fili che aveva messo in evidenza all'inizio con la descrizione del quadro di Velázquez Las hilanderas, a ribadire che esiste uno stretto legame narrativo tra pittura e scrittura e che la scrittura è un "lavoro di tessitura" e "costruisce un elaborato sistema di riflessioni sull'atto stesso della creazione artistica". 
